\title{
Frontières
}

\section{La notion de responsabilité criminelle à l'ère des antidépresseurs}

\section{Christian Saint-Germain}

Volume 21, numéro 2, printemps 2009

Détresse psychique et antidépresseurs

URI : https://id.erudit.org/iderudit/039452ar

DOI : https://doi.org/10.7202/039452ar

Aller au sommaire du numéro

Éditeur(s)

Université du Québec à Montréal

ISSN

1180-3479 (imprimé)

1916-0976 (numérique)

Découvrir la revue

Citer cet article

Saint-Germain, C. (2009). La notion de responsabilité criminelle à l'ère des antidépresseurs. Frontières, 21(2), 15-20. https://doi.org/10.7202/039452ar
Résumé de l'article

Cet article tente de problématiser le paysage éthique inédit issu de l'augmentation de la consommation d'antidépresseurs. La prise de psychotropes modifie les comportements des sujets et leur responsabilité. Faut-il pour autant développer une sensibilité éthique nouvelle pour évaluer des actes posés sous influence ? Le développement des psychotropes, la prolifération des diagnostics de dépression forcent à reconsidérer le degré de responsabilité morale et éventuellement légale des individus modernes. Cet article décrit l'état des lieux lorsque les molécules pèsent sur la conscience des sujets.
Ce document est protégé par la loi sur le droit d'auteur. L'utilisation des services d'Érudit (y compris la reproduction) est assujettie à sa politique d'utilisation que vous pouvez consulter en ligne.

https://apropos.erudit.org/fr/usagers/politique-dutilisation/ 


\section{Résumé}

Cet article tente de problématiser le paysage éthique inédit issu de l'augmentation de la consommation d'antidépresseurs. La prise de psychotropes modifie les comportements des sujets et leur responsabilité. Faut-il pour autant développer une sensibilité éthique nouvelle pour évaluer des actes posés sous influence? Le développement des psychotropes, la prolifération des diagnostics de dépression forcent à reconsidérer le degré de responsabilité morale et éventuellement légale des individus modernes. Cet article décrit l'état des lieux lorsque les molécules pèsent sur la conscience des sujets.

Mots clés: antidépresseurs responsabilité éthique - dépression imputabilité - inflation diagnostique.

\section{Abstract}

This article deals with the ethical issues related to the increasing use of antidepressants. The intake of psychoactive drugs causes a modification of behavior and challenges the level of responsibility derived from it. Is it then necessary to develop new ethical ways to understand actions taken under influence? This article describes the new situation triggered by the growing influence of molecules on the decision-making process.

Keywords: antidepressants - ethical responsibility - depression accountability - inflation diagnosis.

\section{La notion \\ de responsabilité criminelle à l'ère des antidépresseurs}

\author{
Christian Saint-Germain, Ph. D., \\ professeur titulaire, Département de philosophie, \\ Université du Québec à Montréal.
}

L'éthique ne peut faire abstraction de $l^{\prime}$ « histoire du présent » quant à la nature de la subjectivité. Il ne s'agit pas de prétendre à la reconstitution d'une autre "révolution copernicienne» quant à la situation du sujet conscient, mais de faire ressortir l'effet de décentrement produit par la mise en fabrique institutionnelle d'un sujet autrement confectionné par l'ordre social actuel, en un mot de saisir les impacts d'un «malaise dans la subjectivation» (Lebrun, 2008).

Le résultat de la désagrégation des anciens modes structurants de la sociabilisation (famille, école, travail) et de la reconnaissance deviennent d'autant plus constrictifs qu'ils sont passés d'une extériorité d'apparence contractuelle à l'invisibilité d'un acquiescement sans mesure. Il $\mathrm{y}$ a en effet peu de discussion possible avec un effet secondaire ou une action pharmacologique directe, sédative ou stimulante sur l'esprit ou l'humeur d'un sujet.

Autrement dit, par le plus curieux des retournements, le travail de socialisation des individus ne procède plus tant des institutions, de l'ordre symbolique, que de la métabolisation moléculaire depuis un transit pharmaceutique qui va de l'enfance jusqu'à la fin de vie sous sédation. L'effacement des encadrements formels, la désinstitutionnalisation des formes sociales n'aboutit pas à un accroissement des libertés individuelles ou à quelque affranchissement, comme une conscience naïve aurait pu escompter, mais au contraire à une intériorisation plus fine - d'aucuns diraient maligne - des impératifs de la production de soi dans le champ social.

Un autre type d'être humain - de sujet éthique - surgit à la confluence des décombres des institutions traditionnelles et dans la volonté sans objet des individus modernes de s'épanouir sans égard pour la tradition, la transmission de valeurs intergénérationnelles ou afin d'assurer la pérennité de quelque ordre que ce soit.

L'un des événements majeurs dans l'histoire de la subjectivité occidentale est l'apparition discrète, bien que statistiquement croissante, de la consommation à des fins médicales des antidépresseurs. Au Canada, le diagnostic de dépression est en pleine expansion statistique. Santé 
Canada observe qu'entre 1994 et 2004 les demandes de soin concernant cette affection nerveuse ont pratiquement doublé1.

En conséquence, la recrudescence de l'usage de psychotropes marque l'avènement d'un fait social qui propage, établit une norme de confort psychique assimilée, par l'ordre médico-pharmaceutique moderne, à l'équilibre intérieur et à la santé mentale. Une certaine quantité de souffrance individuelle n'est désormais plus socialement acceptable ${ }^{2}$. Évidemment, la nature particulière des inconforts et des vicissitudes psychiques ne peut être complètement mesurée. Il existe toutefois un certain nombre de moyens d'évaluer, d'apprécier objectivement la sévérité du vécu dépressif. La méthode la plus connue est sans doute la mesure d'Aaron Beck, communément appelée "Échelle de Beck» (Ionescu, 2006).

Il n'en demeure pas moins que la «tendance lourde» des sociétés avancées est de minimiser sous le mode de l'analgésie généralisée l'expérience humaine. Ce dispositif pharmacologique dans lequel se reconfigure la subjectivité n'est pas une situation passagère, un "effet de mode», mais le «pli» singulier que notre époque fait prendre à un nombre toujours plus important de sujets. Ce pli est imprimé par le continuum pharmaceutique qui désormais enserre les comportements depuis une reformulation moléculaire de la condition psychique des individus.

Il existe une symétrie déconcertante entre le Prozac et la Ritaline. Le premier est prescrit pour les femmes déprimées manquant d'estime de soi : il leur donne davantage de sentiment du mâle alpha qui accompagne les hauts niveaux de sérotonine. La Ritaline, de son côté, est largement administrée aux jeunes garçons qui ne veulent pas rester tranquilles en classe, parce que la nature ne les a jamais programmés à cette fin. D'un côté comme de l'autre, les deux sexes sont ainsi orientés vers une personnalité androgyne moyenne, satisfaite d'elle-même et socialement conciliante c'est-à-dire le courant «politiquement correct» de la société américaine moyenne. [...] Ces deux psychotropes paraissent bien plus efficaces que la socialisation de l'enfance à l'ancienne et les thérapies bavardes du freudisme ne l'ont jamais été. Leur usage s'est répandu à des millions et des millions de gens à travers le monde, suscitant beaucoup de controverses sur leurs conséquences à long terme pour la santé du corps, mais - curieusement - aucune discussion à propos de ce qu'ils impliquent sur notre compréhension conventionnelle de l'identité et du comportement

moral de l'individu (Fukuyama, 2004, p. 101-102, soulignement de l'auteur).

Sans développer ici spécifiquement les répercussions juridiques (voir SaintGermain, 2007) de ce changement anthropologique majeur, ce sujet d'un genre nouveau apparaît désormais dans des circonstances pharmacologiques particulières. Il n'est plus dans un rapport de "spontanéité» ordinaire avec son action, et donc susceptible d'un jugement d'attribution, mais il est soumis aux lois de la biologie modifiées par la prise d'une substance thérapeutique, médicament ou drogue. Autrement dit, sans être aliéné mental au sens strict de la législation pénale, ni même intoxiqué, le sujet éthique n'est plus «ni tout à fait lui-même ni tout à fait un autre ». Son rapport à l'ipséité ${ }^{3}$, à la continuité d'un moi identique, n'est plus cet état normal qui permet de présumer un comportement habituel.

La licéité d'une prescription d'antidépresseurs distingue radicalement dans une perspective morale ou juridique la conduite du sujet malade de celle d'un consommateur de stupéfiants à des fins récréatives. Dans le cas d'un dépressif, il en va d'une personne qui escompte raisonnablement d'un traitement le soulagement de ses maux et la réintégration dans un milieu de travail. Il s'agit d'une démarche responsable, dans l'autre cas, d'une solution hasardeuse ne serait-ce qu'en raison du degré d'inconnu que représente la composition des substances illégales.

L'un des problèmes posés par la consommation d'antidépresseurs réside dans la prise à long terme, la non-interruption du médicament. L'assuétude développée n'est pas tant physiologique que psychologique. Le fait qu'un nombre croissant d'individus vivent continûment sous influence n'est pas sans conséquence éthique si l'on considère que les molécules antidépressives interviennent principalement sur les centres psychiques qui poussent à agir. Dans ce contexte, la représentation du concept de responsabilité éthique correspond-elle encore à l'expérience contemporaine de la subjectivité?

\section{L'IMPULSION À AGIR}

La capacité à pouvoir apprécier la portée morale d'un acte est ici remise en cause par la médication. La nouvelle donne pharmacologique place devant le contexte suivant: un sujet potentiellement coupable en regard du droit, mais irresponsable d'un point de vue éthique. En effet, on peut très bien être en mesure de distinguer le bien du mal, saisir parfaitement la nature de l'acte que l'on s'apprête à poser sans pour autant avoir la capacité d'inhiber cette action. Comment alors qualifier moralement une action involontaire, qu'on ne pouvait par aucun moyen physiologique empêcher ni même surtout prévoir? Peut-on sérieusement reprocher ses actes à un sujet sous l'emprise d'une impulsion irrésistible, d'une forme nouvelle de dépendance, si ces derniers sont causés ou encore résultent de la prise d'une médication licite?

Tout se passe en regard de l'imputabilité dans la modernité comme si l'expression «responsable, mais pas coupable ${ }^{4}$ était à l'échelle du sujet l'axiome principal d'où dérive le rapport à la loi. On reconnaît dans cette affirmation en modèle réduit le rapport singulier du sujet à la légalité dans l'horizon totalitaire ${ }^{5}$.

Ce débat résume les limites des deux instances productrices de vérité que sont en Occident le droit et la médecine lorsque prolifèrent les diagnostics de dépression. C'est tout le concept de sujet responsable qui est mis à mal par l'usage immodéré des molécules psychotropes et l'incertitude qui vient du «dépistage » d'autant de troubles mentaux par l'autorité médicale ${ }^{6}$.

Le paradoxe subjectif au regard de l'autonomie de la volonté se résume ainsi: un même esprit peut conserver son statut d'agent moral sans être pour autant en mesure de s'abstenir de commettre des actions répréhensibles. C'est là le caractère proprement désinhibiteur de l'influence médicamenteuse. Autrement dit, si l'antidépresseur vise à supprimer les deux symptômes caractéristiques de la dépression, le ralentissement psychomoteur et la tristesse (Widlöcher, 1983, p. 37), il ne peut, s'il est efficace, que provoquer l'accroissement de l'activité d'un sujet prostré. Cependant, tout indique que dans certains cas, l'administration du traitement n'est pas sans risque comme le soulignait Santé Canada dans une mise en garde de 2004 :

Santé Canada avertit les Canadiens que les inhibiteurs sélectifs du recaptage de la sérotonine (ISRS) et d'autres nouveaux antidépresseurs, sont maintenant accompagnés de mises en garde plus vigoureuses. Ces nouvelles mises en garde précisent que les patients de tous âges qui prennent ces médicaments peuvent présenter des changements comportementaux et/ou émotifs pouvant être associés à un risque accru de poser des gestes autodestructeurs ou de faire du mal à autrui.

Les patients, leur famille et leurs soignants doivent savoir qu'un petit nombre de patients prenant ce type de médicaments ont le sentiment que leur état général a empiré plutôt 
que de s'améliorer, en particulier durant les premières semaines de traitement ou lors des ajustements de posologie. Ils peuvent, par exemple, éprouver une sensation inhabituelle d'agitation, des sentiments d'hostilité ou d'anxiété, avoir des pensées impulsives ou troublantes, notamment envisager de poser des gestes autodestructeurs ou de faire du mal à autrui (Santé Canada, 2004).

\section{ÉCLAIR DANS UN CIEL BLEU}

Parmi les dangers comportementaux soulignés par Santé Canada dans sa notice, il arrive qu'un individu fonctionnel puisse ne pas ressentir les modifications progressives de ses états de conscience dues à l'effet discret de la métabolisation d'un médicament. Ce glissement du rapport de soi à soi n'est pas non plus immédiatement perceptible par les proches. Une mutation insoupçonnable du comportement n'attend alors qu'un événement qui déclenche ou révèle la part d'insu recelée dans l'interaction médicamenteuse avec l'environnement. Pareille situation n'implique pas non plus un facteur d'aliénation sévère - dissociation, hallucinations ou toutes autres formes de ces désagréments majeurs.

Agressivité accrue, sentiment d'invincibilité pourraient fort bien être les conséquences de l'absorption d'antidépresseurs, si l'on considère ces médicaments comme des désinhibiteurs de l'action.

Les cas de kleptomanie épisodique, de conduite erratique, de sautes d'humeur à l'égard des proches qui n'appartiennent pas au comportement habituel d'un individu illustrent cette anomalie. Le concept de sujet moral dans la modernité doit être reconsidéré à l'aune de la médicalisation globale, du continuum pharmaceutique qu'emprunte désormais un grand nombre d'individus pour fonctionner adéquatement en société. Il suffit de penser à la prise de Ritalin dès le jeune âge jusqu'à l'usage d'antidépresseurs à l'adolescence puis à l'âge adulte et la fin de vie sous sédation.

Autrement dit, une société qui cherche à sauver l'apparence d'ordre en banalisant la consommation de psychotropes (à travers la multiplication des diagnostics de dépression ou la facilité à obtenir ce type de médicament d'un généraliste) ne peut ensuite tenir rigueur ni feindre d'ignorer les changements dont elle est l'agent. Une campagne de sensibilisation à la dépression n'est jamais sans effet sur l'accroissement des ventes d'antidépresseurs. Comme le constate le sociologue Alain Ehrenberg (1998, p. 292) :
En l'an 2000, les pathologies de la personne sont celles de la responsabilité d'un individu qui s'est affranchi de la loi des pères et des anciens systèmes d'obéissance ou de conformité à des règles extérieures. La dépression et l'addiction sont comme l'avers et l'envers de l'individu souverain, de l'homme qui croit être l'auteur de sa propre vie alors qu'il en reste «le sujet au double sens du mot: l'acteur et le patient».

\section{QUEL SUJET ?}

Que devient le sujet moral moderne dans ce paysage? Comment la consommation massive, légale, d'antidépresseurs par un nombre grandissant d'individus affectet-elle les représentations communes du fonctionnement de l'esprit?

Cette médicalisation du comportement met d'abord en scène un sujet responsable résolu à guérir d'un «trouble mental». À partir du moment où le Manuel diagnostique et statistique des troubles mentaux $D S M-I V$ établit une nosographie «organique » concernant la condition psychique du sujet, il devient difficile d'apprécier le comportement d'une personne selon les mêmes barèmes ou selon des critères moraux habituels. Il ne s'agit pas ici de prendre parti dans les débats entourant la pertinence de la médication psychotrope. Il s'agit plutôt de prendre en compte les effets de la désubjectivation dans ce qui reste de l'édifice doctrinal éthique. Comment concilier le libre arbitre et l'influence déterminante d'une substance qui modifie l'être-au-monde initial?

La question de savoir si le «continent» dépressif existe véritablement ${ }^{7}$, s'il existe en psychiatrie une telle chose que la sûreté du diagnostic, ou si la consommation de médicaments n'est que le résultat d'une inflation du pouvoir publicitaire de commercialisation des médicaments, tout cela n'est pas pertinent à notre étude ${ }^{8}$. La dépression nerveuse est un «fait social» avéré ${ }^{9}$.

\section{UN SUJET ÉTRANGER À LUI-MÊME}

Dans le ralentissement psychomoteur caractérisant l'installation de l'humeur dépressive, les antidépresseurs rétablissent un certain tonus du sujet. Cette relance de l'impulsion vitale pousse parfois au passage à l'acte suicidaire dans les débuts de traitements non supervisés. Ce redoutable effet «secondaire» tient à un déverrouillage de l'activité motrice tenue jusque-là en respect ou annulée par l'hyperactivité de l'esprit souffrant. Les idéations suicidaires demeurent dans les débuts de la prise du médicament, mais la métabolisation des molécules antidépressives engage par la suite une énergie insoupçonnée. Autrement dit, ce qui participait de la torpeur conservatrice du sujet dans l'affect dépressif est renversé par l'orientation impulsive donnée par le médicament ${ }^{10}$.

Ce retournement du psychisme contre lui-même, cette volte-face a conduit des patients sans antécédents criminels à des comportements meurtriers et même suicidaires. Dans une importante étude publiée dans Archives General of Psychiatry (2006), des chercheurs finlandais concluent leur recherche étalée sur 3, 4 années et sur 15390 patients en ces termes: "Parmi les sujets suicidaires qui avaient déjà pris des antidépresseurs, l'usage courant de tout antidépresseur a été associé à une augmentation marquée du risque de tentatives de suicide et en même temps à une diminution marquée de suicides réussis résultant en un décès » (trad. libre, Tiihonen et al., 2006, p. 1358) ${ }^{11}$.

\section{SOCIÉTÉS DÉPRESSIVES \\ ET EFFETS DES PSYCHOTROPES}

Dans l'affinement des modes de contrôle des conduites ${ }^{12}$, et les enjeux nouveaux de la socialisation, les sociétés avancées tendent à déployer une forme "d'orthopédie moléculaire», d'assistance continue aux individus en proie aux mutations anomiques rapides des sociétés. À l'occasion de la multiplication des ordonnances de substances psychotropes, le sujet sous influence correspond à une réalité statistique de plus en plus importante ${ }^{13}$. Les catégories de ces substances comprennent la plupart des antidépresseurs connus sous l'appellation générale des inhibiteurs sélectifs de la recapture de la sérotonine (ISRS) $\left(\right.$ Zoloft $^{\circledR}$, Paxil $^{\circledR}$, Effexor $^{\circledR}$, Luvox $^{\circledR}$, Prozac $^{\circledR}$ ) et certains autres appelés antidépresseurs atypiques ${ }^{14}$ (Desyrel ${ }^{\circledR}, \operatorname{Zyban}^{\circledR}$, Wellbutrin ${ }^{\circledR}$ ). Leurs mécanismes d'action sont résumés en ces termes:

Les antidépresseurs ont pour effet immédiat d'augmenter la concentration de certains neurotransmetteurs dans les synapses. Selon l'antidépresseur en cause, cet effet résulte généralement d'un blocage du recaptage présynaptique d'une ou de plusieurs monoamines biogènes ou d'une inhibition du métabolisme de ces mêmes neuromédiateurs (Léonard et Ben

Amar, 2002, note 53, p. 762).

La consommation massive de psychotropes inaugure un chapitre inédit dans l'histoire des dépendances. Cette mutation $\mathrm{du}$ soi tend à sortir du strict registre de la prescription médicale pour s'affirmer comme technique de maquillage, opération cosmétique complexe. Dépendance confortable, substitution des malaises par l'installation d'un climat propre à 
la subjectivité. "Qu'est-ce alors que la drogue? Un artifice pour fabriquer de l'individu, une chimie de la promotion de soi » (Ehrenberg, 1991, p. 8). L'automédication dissimulée derrière l'élargissement du concept de dépression n'est pas un moyen pris pour résister à la société actuelle mais la tentative désespérée de s'y accommoder.

Cet attachement des sujets à l'espace social constitue le lien même, la soumission à l'interpellation permanente, au devoir de répondre à un environnement de contraintes: travail flexible, horaires brisés, précarité économique, licenciement en masse, stagnation en maison de personnes âgées, compromis de toute nature. Cette école de la sujétion constitue le roc de la castration sociale. État de fait par lequel la subjectivité est avant tout assujettissement. Sa constitution n'est que l'ensemble des moments réifiés de l'exigence sociale. D'où, par la suite, cette disposition à croire que ce qui apparaît sous les traits secourables du soin médical procède nécessairement d'une admirable bienveillance, d'une attention humaniste.

\section{LIBRE ARBITRE ET DÉSINHIBITION MOTRICE DES SUJETS}

La responsabilité éthique repose sur l'acceptation des conséquences laïques de la notion de libre arbitre. Ce concept central est l'une des structures porteuses de toutes les fictions visant à arrimer un sujet à ses actes et à en lui imputer les conséquences. Cette manière de comprendre l'action du sujet présuppose que celle-ci est posée en l'absence de déterminisme, de contraintes neurologiques.

Le principe est simple et efficace au plan de la représentation. Il tient à la chaîne causale suivante: en regard d'une norme ou d'une obligation éventuellement universalisable, chaque sujet éthique est présumé avoir pu, à chacun des moments qui précèdent la commission d'un acte volontaire répréhensible, interrompre l'enchaînement qui conduit à sa réalisation. Comme dans une règle grammaticale, le verbe (l'acte) et le complément (les conséquences) se rapportent «naturellement » au sujet. Pareille approche implique un sujet unifié, une adéquation parfaite de la volonté consciente avec l'agir.

Cette reconstruction rétrospective ne repose pas tant sur la «réalité» du fait fautif que sur l'efficace de la narration qui réussit à établir le plus intimement possible le niveau de connexité entre l'agent, l'acte et les témoins ${ }^{15}$. Elle implique en éthique qu'un sujet puisse s'extraire d'un contexte bureaucratique. Cette méthode d'analyse des conduites tient à un a priori, une présomption indiscutable selon laquelle l'agent dispose des moyens de s'interrompre, de délibérer intérieurement, et possiblement de prendre une décision contraire à chacune des étapes de son action. Dans cette perspective cinématographique, il suffit de repasser le «film» des événements à l'envers pour découvrir la genèse de l'intention initiale. Un «dedans» psychique se révèle complètement dans la patence d'un «dehors » fautif ${ }^{16}$. Or, pour très utile que puisse être cette mise en forme du processus incriminateur, elle n'en demeure pas moins une fiction culpabilisatrice en comparaison de la complexité des représentations philosophiques contemporaines et de notions telles que la conscience, la volonté, l'intention.

Il est difficile de concevoir que l'on puisse en éthique encore tenir pour avérée une représentation de la subjectivité humaine grevée par une conception de la liberté humaine héritière d'Aristote ou de Kant.

Pour Kant, la masturbation est une faute morale plus grave que le suicide. Il en prend pour preuve le fait «que l'on tient même pour immoral d'appeler un tel vice par son nom » (et il fait bien attention d'ailleurs à ne pas commettre cette faute morale lui-même) alors que personne n'hésite à blâmer ouvertement le suicide en le nommant (Ogien, 2007, p. 42).

L'éthique telle qu'elle est encore enseignée conserve des conceptions de l'intériorité d'une naïveté épistémologique prémoderne lorsque apparaît un sujet autrement configuré, un sujet sous influence. Surtout si l'on apprécie la notion de libre arbitre à partir de sa fragile vraisemblance optique tout entière contenue dans la découpe de l'illusion narrative suivante:

L'affirmation du libre arbitre relève de l'illusion symbolique [...], car il s'agit, à partir d'une situation que nous occupons réellement, de nous projeter en des situations que nous n'occuperons jamais, puisque: ou bien nous nous imaginons fictivement dans le passé en position où la situation que nous occupons actuellement apparaît symboliquement comme un simple possible, ou bien nous nous projetons en des situations qui ne seront jamais réelles dans la mesure où elles ne peuvent nous apparaître comme ayant quelque réalité que du point de vue de cette situation que nous occupons et qui ne sera jamais plus la même (Cléro, 2001, p. 22).

Ce travelling vers la situation d'un auteur anonyme compris dans le texte d'une éthique conséquentialiste fait partie de toute la charpente des délégations qui permettent le montage de l'illusion perceptuelle nécessaire au faisceau culpa- bilisateur. En fait, la responsabilité d'un sujet n'apparaît jamais mieux ou ailleurs que dans l'efficace d'un récit bien raconté passé dans le goulot de l'entonnoir d'une suite ininterrompue de restrictions mentales. La confusion constante entre une position subjective et objective, entre une interprétation qui découle d'une reconstitution rétrospective et un principe moral abstrait n'est pas le moindre des écueils. Se référant à David Hume, Jean-Pierre Cléro observe que :

[...] [L]orsqu'on nous conteste la prétention [de la soumission de nos actions à notre volonté], invités à la mettre à l'épreuve, nous sentons alors que la volonté se dirige sans peine en tout sens et qu'elle produit d'elle une image, même du côté où elle ne réside pas réellement. Nous nous persuadons que cette image ou que ce mouvement fictif aurait pu s'accomplir dans la réalité même; parce que, cela dût-il être contesté, nous trouvons, lors d'un second essai que c'est possible. Il est en effet possible que je puisse faire, dans la situation présente, ce que je n'ai pas fait dans la situation précédente : cela prouve-t-il que j'avais toute liberté de le faire? (Cléro, 2001, note 62 , p. 23.)

La psychologie, la philosophie de l'esprit tendent à douter, voire à remettre en cause la prétention même selon laquelle il existerait une telle "chose» que la prééminence de la conscience ${ }^{17}$, le libre arbitre ${ }^{18}$ qui détermine tout autant l'unicité de la volonté qu'il régule la formation d'une intention ${ }^{19}$. La question de la sensibilité aux conceptions implicites du fonctionnement du cerveau humain dans l'évaluation des conduites revêt une importance cruciale lorsque s'ajoutent, à cette complexité initiale, des familles de médicaments qui en modifient l'état ${ }^{20}$.

\section{L'IMPUTABILITÉ EN REGARD DES MOUVEMENTS D'HUMEUR}

Tout ce qui arrive en tant qu'unité à la conscience est déjà monstrueusement compliqué: nous n'avons jamais qu'une apparence d'unité.

(Nietzsche, cité dans Wotling, 1999,

p. 31.)

Sans rapport direct avec les situations d'intoxication volontaire ou même d'automatisme psychiatrique, le sujet sous l'emprise des psychotropes, celui qui fait usage d'antidépresseurs obtenus par l'entremise de prescriptions médicales se trouve, dans chacune de ses actions, placé dans une situation inédite. Si l'on tient compte de l'influence des psychotropes, de l'idiosyncrasie de chaque sujet sous influence, ceuxci ne seraient plus, à proprement parler, 
imputables. Le philosophe Paul Ricoeur définit l'imputabilité en ces termes: [...] [L]'idée de rendre compte putare, computare; imputer en son sens le plus général, c'est mettre en effet sur le compte de quelqu'un une action blâmable, une faute, donc une action confrontée au préalable à une obligation ou à une interdiction que cette action enfreint. [...] L'imputabilité est la capacité d'être tenu pour responsable de ses actes comme en étant leur véritable auteur (Ricoeur, 2001, p. 96-97).

La modernité bouleverse la nature même de la compréhension de ce qu'est la responsabilité personnelle. L'effet conjugué de la déstructuration des institutions et du climat d'intense sollicitation remet en cause la capacité pour un sujet de s'appartenir complètement. Tout le rapport de la subjectivité à l'appareil moteur, au rapport de soi à soi, à l'exercice de la volonté subit la force des contraintes moléculaires censées redresser ou empêcher l'appesantissement dépressif. À partir du moment où un psychotrope fonctionne, il implique rien de moins que l'effacement progressif du ralentissement psychomoteur, l'autodénigrement, l'insomnie ou l'hypersomnie, et éventuellement les idéations suicidaires.

Modifier l'humeur ${ }^{21}$ d'un sujet n'a rien d'une opération superficielle. Il en va d'une intervention somatique sur ce qui correspondait à la trame narrative, à l'intériorité de soi naguère comprise depuis le registre de la plainte adressée à autrui, de l'histoire racontée. Ce moment où s'exerçait la récollection narrative cède ici sa place à une perspective biologique qui, progressivement, gomme l'historicité ou la singularité du symptôme particulier pour le soumettre à l'économie générale de l'augmentation du taux de sérotonine dans le plasma sanguin et sur les sites de récepteurs synaptiques censés commander les mécanismes fondamentaux d'adaptation du sujet à son environnement.

\section{LE SUJET INSTABLE}

L'expérience de la modernité place le sujet au carrefour de champs d'influence divers, qu'il s'agisse des médias, des produits pharmaceutiques, de la déstructuration des formes institutionnelles (familles, travail, écoles) qui marquaient encore récemment une certaine stabilité en regard de la vie sociale. Le philosophe Gilles Deleuze ne manquait pas d'observer que:

Réformer l'école, réformer l'industrie, l'hôpital, l'armée, la prison; mais chacun sait que ces institutions sont finies, à plus ou moins longue échéance. Il s'agit seulement de gérer leur agonie et d'occuper les gens, jusqu'à l'installation de nouvelles forces qui frappent à la porte. Ce sont les sociétés de contrôle qui sont en train de remplacer les sociétés disciplinaires (Deleuze, 1990, p. 241).

Ce serait manquer à la plus élémentaire probité intellectuelle en éthique que de ne pas reconsidérer la position subjective dans cet environnement incertain ${ }^{22}$. Le statut de l'enfant hyperactif précocement placé sous médication comme celui de l'adulte dépressif redessine le rapport de chacun de ces sujets à l'intentionnalité. Tout se passe comme si les sociétés de consommation avancée suscitaient - notamment pour les exigences nouvelles du monde du travail une subjectivité encline à d'irrésistibles impulsions, continuellement mobilisée, attentive jusqu'au point de rupture psychique. Ce contexte exige de considérer la décentration d'un sujet par rapport à ses actes qui ne soient pas spontanément assimilables aux catégories de la négligence ni même à celles, plus anciennes, de fautes morales. Le sujet moderne constamment soumis à des stratégies d'accaparement vit sous la contrainte jusqu'au vertige. $\mathrm{Ni}$ marionnette ni automate, un sujet sous influence tel qu'il est décrit par Françoise Proust en ces termes:

À chaque instant la conscience moderne est bombardée de données sans suite ni consécution: automatisations et morcellements des activités, prostitution des biens et des personnes en marchandises, atomisation des masses, rafale d'informations [...] Le monde a déclaré la guerre à la conscience. Traumatisée, soumise à une série incessante de chocs, à un déferlement d'agressions, elle n'est plus en mesure de faire face et de dominer ses objets. Elle, "dont le rôle est de protéger des sensations », se voit débordée. [...] Ainsi, la conscience est bien marquée, mais elle n'est que cela : marquée, balafrée, signée, soufflée. Ce qui la marque ce n'est pas le présent ou le souvenir du contenu d'une expérience, mais justement une simple marque, une simple balafre, une simple encoche: comme un cratère dans un champ, une vitre soufflée dans une façade ou une amputation d'organe (Proust, 1994, p. 21-22).

\section{Bibliographie}

ANDLER, D. (1992). Introduction aux sciences cognitives, Paris, Gallimard.

BRÉMOND, M. ET A. GÉRARD (1998). Vrais déprimés, fausses dépressions, Paris, Flammarion.

CLÉRO, J.-P. (2004). Les raisons de la fiction: les philosophes et les mathématiques, Paris, Armand Colin.
CLÉRO, J.-P. (2001). Déterminisme et liberté, Paris, Ellipses.

DELEUZE, G. (1990). Pourparlers, Paris, Éditions de Minuit.

EHRENBERG, A. (1998). La fatigue d'être soi. Dépression et société, Paris, Odile Jacob.

EDELMAN, G.M. (1992). Biologie de la conscience, Paris, Éditions Odile Jacob.

FUKUYAMA, F. (2004). La fin de l'homme. Les conséquences de la révolution biotechnique, Paris, Gallimard.

GOUX, J.-J. (1978). Les iconoclastes, Paris, Seuil.

HAAR, M. (1983). "La critique nietzschéenne de la subjectivité », dans Genèse de la conscience moderne, R. ELLRODT (dir.), Paris, Presses universitaires de France.

IONESCU, S. (2006). 14 approches de la psychopathologie, Paris, Armand Colin.

IMS HEALTH CANADA (2005). " Growth in retail prescriptions slows in 2004 », en ligne, <http://www.imshealthcanada.com/ web/content/0,3148,77303623_6387 2702_77770096_77809159,00.html>, consulté le 2007-12-20.

LEBRUN, J.-P. (2001). Les désarrois nouveaux du sujet. Prolongements théoricocliniques au monde sans limite, Paris, Érès.

LÉONARD, L. ET M. BEN AMAR, (2002). Les psychotropes. Pharmacologie et toxicomanie, Montréal, Presses de l'Université de Montréal.

OGIEN, R. (2007). L'éthique aujourd'hui. Maximalistes et minimalistes, Paris, Gallimard.

PIGNARRE, P. (2001). Comment la dépression est devenue une épidémie, Paris, Éditions de la Découverte.

PROUST, F. (1994). L'histoire à contretemps. Le temps historique chez Walter Benjamin, Paris, Éditions du Cerf.

REDEKER, R. (2007). Dépression et philosophie. Du mal du siècle au mal de ce siècle, Paris, Éditions Pleins Feux.

RICOEUR, P. (2001). Le juste 2, Paris, Éditions Esprit.

RICOEUR, P. (1990). Soi-même comme un autre, Paris, Seuil.

RYLE, G. (1978) La notion d'esprit (The Concept of Mind). Pour une critique des concepts mentaux, Paris, Payot.

SAINT-GERMAIN, C. (2007). "L'imputabilité en droit criminel canadien des comportements induits par les antidépresseurs ", Revue de droit de l'Université de Sherbrooke, vol. 38, no 1, p. 167-214.

SAINT-GERMAIN, C. (2005). Paxil ${ }^{\circledR}$ Blues, Antidépresseur: La société sous influence, Montréal, Éditions du Boréal.

SANTÉ CANADA (2004). "Santé Canada avertit les Canadiens de la présence de mises en garde plus vigoureuses concernant les ISRS et d'autres nouveaux antidépresseurs ", en ligne, <http://www.hc-sc.gc.ca/ahc-asc/ media/advisories-avis/_2004/2004_31-fra. php>, 3 juin, consulté le 2007-12-20. 
TIIHONEN, J. et al., (2006). «Antidepressants and the risk of suicide, attempted suicide, and overall mortality in a nationwide cohort», Archives General of Psychiatry, vol. 63, p. 1358-1367.

WIDLÖCHER, D. (1983). Les logiques de la dépression, Paris, Fayard.

WOTLING P. (1999), La pensée du soussol. Lire Nietzsche: statut et structure de la psychologie dans la philosophie de Nietzsche, Paris, Allia.

\section{Notes}

1. Au Canada, la dépression est le diagnostic effectué en cabinet médical qui augmente le plus rapidement. De 1991 à 2004, le nombre de visites chez le médecin pour dépression a presque doublé. La majorité des visites (57\%) ayant pour cause la dépression ont été faites par des Canadiens âgés entre 40 et 64 ans (voir IMS Health Canada, 2005).

2. Comme le souligne Robert Redeker (2007, p. 14) : «ainsi, la norme évolue: ce qui physiologiquement peut paraître normal à certaines époques, du fait probablement qu'il est impossible de s'y opposer, peut devenir maladif à des époques suivantes. Donnons un exemple de ce jeu de la norme physiologique: normales naguère encore, et même sacralisées, les souffrances de l'accouchement paraissent aujourd'hui nettement moins acceptables. Ces souffrances cheminent sur la voie de leur dénormalisation ».

3. Sur la question de l'ipséité, lire Paul Ricoeur (1990, p. 198).

4. Formule rendue célèbre par la politicienne française Georgina Dufoix à l'occasion du procès sur le sang contaminé.

5. La réponse «responsable, mais pas coupable» sied parfaitement au moyen de défense, à la position juridique d'Adolph Eichmann à Nuremberg.

6 . Pour interpréter le sens de cette inflation diagnostique, voir Saint-Germain (2005).

7. Voir à ce propos Brémond et Gérard (1998).

8. Cette question a été abordée dans SaintGermain (2005).

9. «En 1970, il y avait cent millions de déprimés dans le monde. Trente ans plus tard, ils sont peut-être un milliard! Selon plusieurs rapports d'organismes officiels, la dépression est aujourd'hui la quatrième cause mondiale de handicap et elle devrait passer au deuxième rang dans les vingt-cinq prochaines années [...] En France, le nombre de patients déprimés et soignés a augmenté de 1 million en dix ans (1980-1991), les femmes étant toujours trois fois plus nombreuses que les hommes. Cela représente une augmentation de $60 \%$. Selon l'Organisation mondiale de la santé (OMS), la dépression sera ainsi dans les prochaines années un des deux grands problèmes de santé publique et peut-être même le premier, avant les maladies cardio-vasculaires » (Pignarre, 2001, p. 11).

10. Ce changement brutal de comportements des patients a d'ailleurs amené non seulement Santé Canada mais la FDA à exiger des fabricants qu'ils publient des mises en garde spécifiques notamment à l'intention des consommateurs adolescents.

11. «Among suicidal subjects who had ever used antidepressants, the current use of any antidepressants was associated with a markedly increased risk of attempted suicide and, at the same time, with a markedly decreased risk of completed suicide and death» (Tiihonen et al., 2006, p. 1358).

12. Il suffit de penser ici à la multiplication des prescriptions de la Ritaline pour exercer un contrôle sur l'enfant d'âge scolaire.

13. «La dépression est le trouble psychiatrique le plus commun chez l'adulte et peut toucher $13 \%$ à $20 \%$ de la population (Finley, Laird et Benefield, 2001). Elle est deux fois plus fréquente chez la femme que chez l'homme (Kamil, 1996; APA, 2000). Aux États-Unis, la prévalence de la dépression majeure à un moment ou un autre de la vie est de $12,7 \%$ chez l'homme et de 21,3\% chez la femme (Kessler et al., 1994). Elle peut débuter à n'importe quel âge et de 50 à $60 \%$ des individus qui en ont souffert manifestent des rechutes (APA, 2000) » (Léonard et Ben Amar, 2002, p. 754).

14. «Ces produits sont considérés comme atypiques, car ils ont un profil pharmacologique particulier qui les distingue des autres types d'antidépresseurs et ils ne présentent pas les mêmes caractéristiques fonctionnelles et structurales que les autres catégories de médicaments (Kamil, 1996). Ils comprennent le bupropion $\left(\right.$ Zyban $^{\circledR}$, Wellbutrin $\left.{ }^{\circledR}\right)$, la néfazodone (Serzone-5ht ${ }^{\circledR}$ ) et la trazodone (Desyrel $\left.^{\circledR}\right)$. La néfazodone et la trazodone agissent principalement sur la transmission sérotoninergique, alors que l'action du bupropion s'exerce surtout sur la transmission dopaminergique (Baldessarini, 2001) " (Léonard et Ben Amar, 2002, note 53, p. 760).

15. «[...] [D]ès qu'un témoin oculaire, [dit] la vérité, raconte ce qu'il a vu à un autre, son témoignage se dégrade aussitôt par le simple fait que ce message est reçu avec une interprétation de la part de l'auditeur et qu'il tend à n'avoir plus de valeur en passant d'un auditeur à un autre»(Cléro, 2004, p. 209). Ce phénomène de dégradation obéit à la Loi de Craig qui s'énonce de la manière suivante: "Loi qui décrit comment se comporte la force probante d'un témoignage, lorsqu'il est repris un certain nombre de fois. À supposer qu'on puisse créditer le premier témoignage d'une probabilité de $\mathrm{a} / \mathrm{b}$, et qu'il soit raconté avec la même force de $a / b$, sa probabilité décroît graduellement de $\mathrm{a} / \mathrm{b}$ à $\mathrm{a}^{2} / \mathrm{b}^{2}$ » (Cléro, 2004, p. 622).

16. Malheureusement, l'élément interne, soit le "dedans » psychique qui fait l'objet de toutes les attentions de la morale autant que du droit criminel, n'est que l'illusion métaphorique que produit l'idée de mécanisme imprudemment décalqué des lois physiques et appliqué aux opérations psychiques. Lintention serait la doublure interne, le présupposé absolu de l'action extérieure. Le philosophe oxfordien Gilbert Ryle constate que : «froncer intentionnellement les sourcils ne consiste ni à faire deux choses dont l'une a lieu sur le front et l'autre dans un endroit (au sens métaphorique du terme) différent, ni à faire une chose avec les muscles de son front et une autre avec quelque organe non corporel. Plus précisément, cela ne consiste pas dans l'exercice préalable de quelque pseudo-muscle occulte qui causerait le froncement de sourcils. "Il a intentionnellement froncé les sourcils" ne relate donc pas deux épisodes. Cet énoncé rapporte un seul épisode, très différent de celui auquel renvoie l'énoncé "il a involontairement froncé les sourcils"; photographiquement, néanmoins, les deux froncements peuvent être identiques» (Ryle, 1978, p. 72).

17. À propos de la conscience, à la suite des observations nietzschéennes, Michel Haar note ceci: "alors qu'elle croit donner des ordres, elle ne fait qu'enregistrer, qu'exécuter. Elle attribue les différents états psychiques à une cause unique, elle-même. [...] Elle croit même - illusion suprême - qu'elle est un substrat qui "contrôle" tout le sujet, détermine et guide jusqu'à la bonne marche des fonctions corporelles» $(1983$, p. 341).

18. «La question du libre-arbitre, elle-même un agglomérat de problèmes enchevêtrés et, pour la plupart, mal posés, dérive partiellement de cette extension inconsciente du terme "volontaire" et des applications fallacieuses des expressions "pourrait" et "aurait pu s'empêcher" qui s'ensuivent» (Ryle, 1978, note 60, p. 72-73).

19. «Dans certaines discussions philosophiques sur le caractère volontaire ou involontaire des actions [...] les termes "volontaires", "involontaire" et "responsable" sont utilisés, non dans leur sens restreint qui concerne les erreurs, réelles ou apparentes, mais dans un sens élargi, englobant toute activité susceptible d'être jugée plus ou moins favorablement selon n'importe quel critère d'excellence ou de conformité. [...] [P] our sauver le droit à utiliser des concepts d'évaluation, il fallait montrer que leur champ d'application se situait ailleurs que dans le monde extérieur; c'est alors qu'on a pensé qu'un monde intérieur de forces non mesurables mais pourvues de fins remplirait cet office » (Ryle, 1978, note 60 p. 72-73).

20. Un pan immense de la philosophie actuelle de l'esprit tend à reconsidérer tous les préjugés moraux qui gouvernaient «l'ancienne psychologie». Voir notamment à ce propos Andler (1992) et Edelman (1992).

21. L'humeur ou thymie est une disposition affective régissant les émotions et les instincts d'un individu. Sa définition selon Delay est classique: "L'humeur est cette disposition affective fondamentale riche de toutes les instances émotionnelles et instinctives qui donne à chacun de nos états d'âme une tonalité agréable ou désagréable, oscillant entre les deux pôles extrêmes du plaisir et de la douleur» (Léonard et Ben Amar, 2002, note 53 , p. 752 ).

22. «Le sujet n'est pas mort; il a perdu sa psychologie restreinte, sa figure familière, il est devenu pulsion, opération, productivité; machine, émetteur-récepteur, réseau, système synaptique enfoncé dans le clignotement des interconnexions sociales et biologiques " (Goux, 1978, p. 122). 